\title{
CRYSTAL STRUCTURE OF (N-N'-BIS(3-ETHOXYSALICYLIDENE)-1,2- DIAMINOPROPANE
}

M.A. Vázquez- Fernández, M.I. Fernández-García, B. Fernández, A. M Gonzalez-Noya, Marcelino Maneiro, M.J. Rodríguez-Doutón

Dpto. de Química Inorgánica. Facultad de Ciencias. Univ. de Santiago de Compostela. Lugo 27002 (Spain).

\begin{abstract}
Three hexadentate Schiff base ligands were obtained by condensation of 3-ethoxy-2hydroxybenzaldehyde and different diamines (1,2-diaminoethane, 1,2-diaminopropane, 1,2-diamino2-metylpropane) to yield $\mathrm{H}_{2} \mathrm{~L}^{\mathrm{n}}\left(\mathrm{H}_{2} \mathrm{~L}^{1}, \mathrm{H}_{2} \mathrm{~L}^{2}\right.$ and $\mathrm{H}_{2} \mathrm{~L}^{3}$, respectively). The ligands have been characterised by elemental analysis, IR, and ${ }^{1} \mathrm{H}$ and ${ }^{13} \mathrm{C}$ NMR spectroscopic techniques, mass spectrometry (ES) and X-ray diffraction.
\end{abstract}

\section{INTRODUCTION}

Our interest in this kind of dicompartmental ligands derives from the known ability of ligands containing six potencial donor atoms to stabilise high oxidation states of metal ions. These ligands present adequate conditions to be useful in the synthesis of octahedral complexes, occupying the equatorial positions around the metal centre. In previous papers we reported the synthesis, structures and properties of Mn (III) complexes with Schiff base ligands ${ }^{1}$. At this goal we have designed the dianionic ligands, $\mathrm{H}_{2} \mathrm{~L}^{\mathrm{n}}$, that contain an inner site with $\mathrm{N}$ - and $\mathrm{O}$ - donor atoms (two imine nitrogen and two phenol oxygen atoms) and an outer coordination site with four $\mathrm{O}$ - donor atoms (two phenoxo and two ethoxy groups).

\section{RESULTS AND DISCUSSION}

The hexadentate Schiff bases, $\mathrm{H}_{2} \mathrm{~L}^{\mathrm{n}}$ were prepared ${ }^{2}$ by condensation of the 3-ethoxy-2hydroxybenzaldehyde with the appropriate diamine in methanol. This mixture was heated at reflux in a round-bottomed flask fitted with a Dean Stark trap to remove the water produced during the reaction. After heating for $3 \mathrm{~h}$, the solution was concentrated to yield a yellow solid. The product was collected by filtration, washed with diethyl ether and dried in air. Yellow crystals of $\mathrm{H}_{2} \mathrm{~L}^{2}$, suitable for single crystal X-ray diffraction studies, were obtained by slow evaporation of methanol solution of the ligand. The characteristiques of the ligands are :

Ligand $\mathrm{H}_{2} \mathrm{~L}^{1}$ : M.p. $144{ }^{\circ} \mathrm{C}$. Anal. Calc. for $\mathrm{C}_{20} \mathrm{H}_{24} \mathrm{~N}_{2} \mathrm{O}_{4}$ : C, 67.4; H, 6.8; N, 7.9. Found: C, 67.2; H, 6.9; N, 7.9\%. MS ES (m/z): 357; IR (KBr, cm $\left.{ }^{-1}\right): v(\mathrm{O}-\mathrm{H}) 3448, v(\mathrm{C}=\mathrm{N}) 1633, v(\mathrm{C}-\mathrm{O}) 1250 .{ }^{1} \mathrm{H}$ NMR $\left(\right.$ DMSO-d $\left._{6}, \mathrm{ppm}\right): \delta 8.54(\mathrm{~s}, 2 \mathrm{H}), 6.74-6.99(\mathrm{~m}, 6 \mathrm{H}), 4.05(\mathrm{c}, 4 \mathrm{H}), 3.92(\mathrm{t}, 4 \mathrm{H}), 1.30(\mathrm{t}, 6 \mathrm{H}) .{ }^{13} \mathrm{C} \mathrm{NMR}$ $\left(\mathrm{DMSO}_{6}, \mathrm{ppm}\right): \delta 167.7(\mathrm{C}=\mathrm{N}), 147.8\left(\mathrm{C}-\mathrm{OCH}_{2} \mathrm{CH}_{3}\right), 152.6(\mathrm{C}-\mathrm{OH}), 58.9\left(\mathrm{CH}_{2}\right)$.

Ligand $\mathrm{H}_{2} \mathrm{~L}^{2}$ : M.p. $91{ }^{\circ} \mathrm{C}$. Anal Calc. for $\mathrm{C}_{21} \mathrm{H}_{26} \mathrm{~N}_{2} \mathrm{O}_{4}$ : C, 68.0; H, 7.0; N, 7.6. Found: C, 67.7; H, 6.8; N, 7.6\%. MS ES (m/z): 371; IR (KBr, cm $\left.{ }^{-1}\right): v(\mathrm{O}-\mathrm{H}) 3446, v(\mathrm{C}=\mathrm{N}) 1632, v(\mathrm{C}-\mathrm{O}) 1248 .{ }^{1} \mathrm{H}$ NMR $\left(\right.$ DMSO-d $\left._{6}, \mathrm{ppm}\right): \delta 8.53 / 8.50(\mathrm{~s}, 2 \mathrm{H}), 6.72-6.97(\mathrm{~m}, 6 \mathrm{H}), 4.02(\mathrm{~m}, 4 \mathrm{H}), 3.99 / 3.75(\mathrm{~m}, 2 \mathrm{H}), 1.36(\mathrm{~m}$, $3 \mathrm{H}), 1.31(\mathrm{~s}, 6 \mathrm{H}) .{ }^{13} \mathrm{C}$ NMR (DMSO-d $\left.{ }^{6}, \mathrm{ppm}\right): \delta$ 167.8/165.9 $(\mathrm{C}=\mathrm{N}), 147.7\left(\mathrm{C}-\mathrm{OCH}_{2} \mathrm{CH}_{3}\right), 152.4(\mathrm{C}-$ $\mathrm{OH}), 20.6\left(\mathrm{CH}_{3}\right)$.

Ligand $\mathrm{H}_{2} \mathrm{~L}^{3}$ : M.p. $88^{\circ} \mathrm{C}$. Anal Calc. for $\mathrm{C}_{22} \mathrm{H}_{28} \mathrm{~N}_{2} \mathrm{O}_{4}$ : C,68.7; H, 7.3; N, 7.2. Found: C,68.5; H, 6.9; N,7.2\%. MS ES (m/z): 385; IR $\left(\mathrm{KBr}, \mathrm{cm}^{-1}\right): v(\mathrm{O}-\mathrm{H}) 3435, v(\mathrm{C}=\mathrm{N}) 1628, v(\mathrm{C}-\mathrm{O}) 1254 .{ }^{1} \mathrm{H}$ NMR $\left(\mathrm{DMSO}_{6}, \mathrm{ppm}\right): \delta 8.54 / 8.51(\mathrm{~s}, 2 \mathrm{H}), 6.70-7.04(\mathrm{~m}, 6 \mathrm{H}), 4.00(\mathrm{~m}, 4 \mathrm{H}), 3.74(\mathrm{~s}, 2 \mathrm{H}), 1.35(\mathrm{~s}, 6 \mathrm{H})$, $1.31(\mathrm{~s}, 6 \mathrm{H}) .{ }^{13} \mathrm{C}$ NMR (DMSO-d $\left.{ }^{6}, \mathrm{ppm}\right): \delta 167.9 / 163.3(\mathrm{C}=\mathrm{N}), 147.8\left(\mathrm{C}-\mathrm{OCH}_{2} \mathrm{CH}_{3}\right), 152.4(\mathrm{C}-\mathrm{OH})$, $25.7\left(\mathrm{CH}_{3}\right)$. 
The crystal structure of $\mathrm{H}_{2} \mathrm{~L}^{2}$, with the numbering scheme, is shown in figure 1. Selected bond lengths and angles, as well as potential hydrogen bonds and bonding scheme, are listed in table 1 . The crystal structure reveals that $\mathrm{H}_{2} \mathrm{~L}^{2}$ exists as discrete molecules. The $\mathrm{C}(11)-\mathrm{N}(1)$ and $\mathrm{C}(41)-\mathrm{N}(4)$ distances of 1.2717(18) and 1.2733(17) $\AA$ respectively, are consistent with a $\mathrm{C}=\mathrm{N}$ double bonding of the imine groups. The two oxygen $\mathrm{O}(130)$ and $\mathrm{O}(430)$ atoms are forming phenolic groups show $\mathrm{C}-\mathrm{O}$ distances $\mathrm{O}(130)-\mathrm{C}(13)$ of $1.3516(16)$ and $\mathrm{O}(430)-\mathrm{C}(43)$ of $1.3486(15) \AA$, according to the expected single bonds.

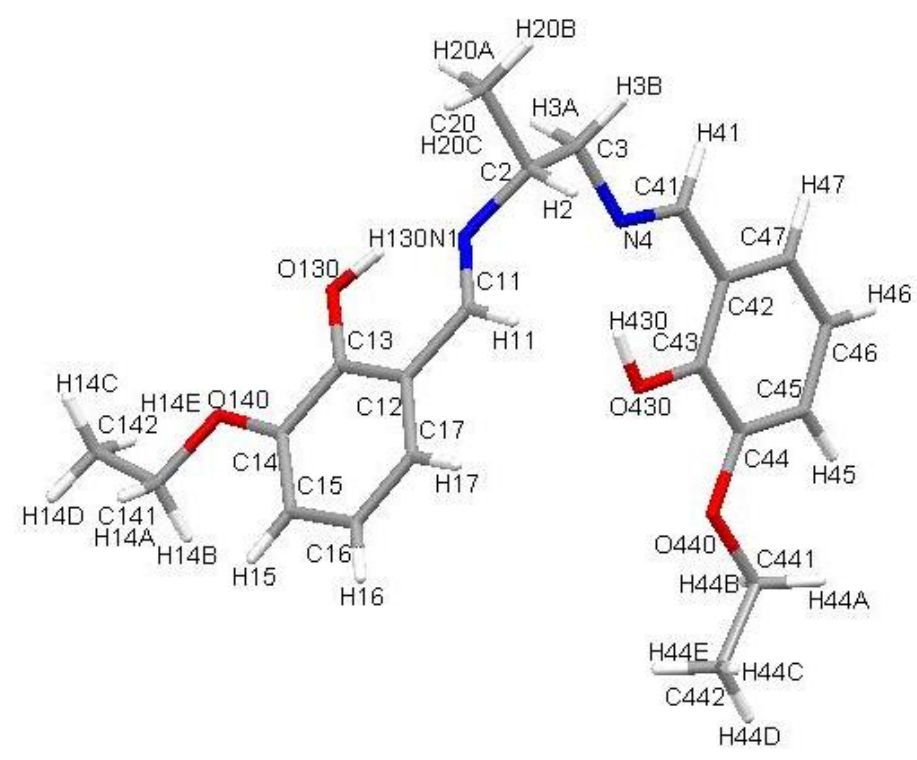

Figure 1: Crystal structure of $\mathrm{H}_{2} \mathrm{~L}^{2}$.

Intermolecular hydrogen bonds exist between imine nitrogen atoms $\mathrm{N}(1)$ and $\mathrm{N}(4)$ and their neighbouring phenolic oxygen atoms $\mathrm{O}(130)$ and $\mathrm{O}(430)$ (see Figure 2 and table 2).

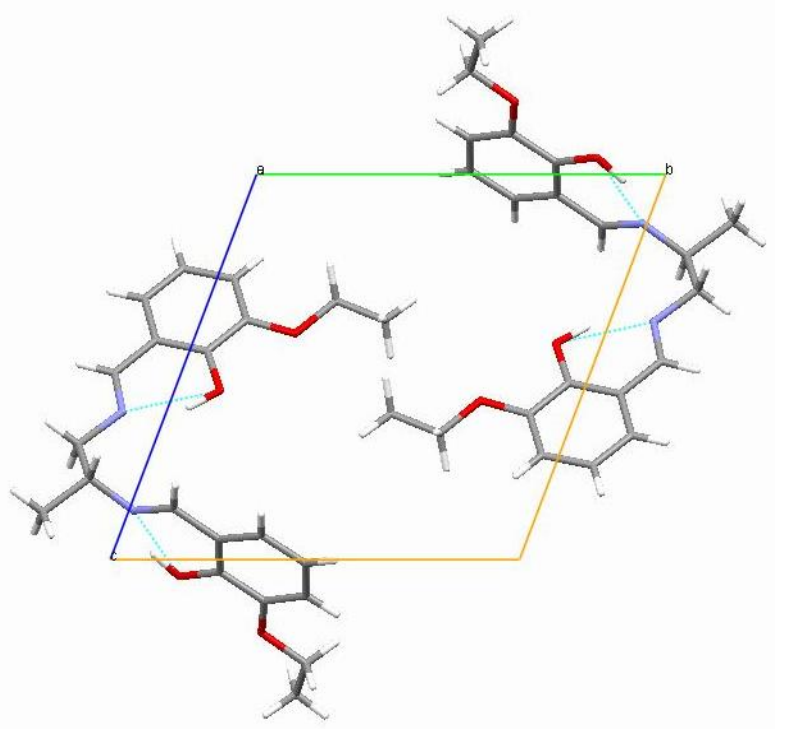

Figure 2: Packing and intermolecular hydrogen bonds of $\mathrm{H}_{2} \mathrm{~L}^{2}$ 
Table 1: Selected bond lengths $(\AA \hat{)})$ and angles $\left(^{\circ}\right)$ for $\mathrm{H}_{2} \mathrm{~L}^{2}$

\begin{tabular}{cccc}
\hline $\mathrm{N}(1)-\mathrm{C}(11)$ & $1.2717(18)$ & $\mathrm{N}(4)-\mathrm{C}(41)$ & $1.2733(17)$ \\
$\mathrm{N}(1)-\mathrm{C}(2)$ & $1.4635(17)$ & $\mathrm{C}(3)-\mathrm{N}(4)$ & $1.4631(17)$ \\
$\mathrm{C}(13)-\mathrm{O}(130)$ & $1.3516(16)$ & $\mathrm{C}(43)-\mathrm{O}(430)$ & $1.3486(15)$ \\
$\mathrm{C}(14)-\mathrm{O}(140)$ & $1.3645(16)$ & $\mathrm{C}(44)-\mathrm{O}(440)$ & $1.3682(16)$ \\
$\mathrm{O}(140)-\mathrm{C}(141)$ & $1.4412(17)$ & $\mathrm{C}(441)-\mathrm{O}(440)$ & $1.4354(16)$ \\
$\mathrm{O}(130)-\mathrm{H}(130)$ & $0.97(2)$ & $\mathrm{O}(430)-\mathrm{H}(430)$ & $0.94(2)$ \\
$\mathrm{C}(11)-\mathrm{H}(11)$ & $0.978(15)$ & $\mathrm{C}(45)-\mathrm{H}(45)$ & 0.95 \\
& & & \\
$\mathrm{C}(11)-\mathrm{N}(1)-\mathrm{C}(2)$ & $119.28(12)$ & $\mathrm{C}(41)-\mathrm{N}(4)-\mathrm{C}(3)$ & $117.73(12)$ \\
$\mathrm{N}(1)-\mathrm{C}(2)-\mathrm{C}(3)$ & $108.50(11)$ & $\mathrm{N}(4)-\mathrm{C}(3)-\mathrm{C}(2)$ & $111.51(12)$ \\
$\mathrm{C}(14)-\mathrm{O}(140)-\mathrm{C}(141)$ & $116.43(11)$ & $\mathrm{C}(44)-\mathrm{O}(440)-\mathrm{C}(441)$ & $118.10(10)$ \\
$\mathrm{O}(130)-\mathrm{C}(13)-\mathrm{C}(14)$ & $118.59(12)$ & $\mathrm{O}(430)-\mathrm{C}(43)-\mathrm{C}(44)$ & \\
\hline
\end{tabular}

Table 2: Hydrogen bonds

\begin{tabular}{ccccc}
\hline $\mathrm{D}-\mathrm{H} \cdots \mathrm{A}$ & $\mathrm{D}-\mathrm{H}(\AA)$ & $\mathrm{H} \cdots \mathrm{A}(\AA)$ & $\mathrm{D} \cdots \mathrm{A}(\AA)$ & $\mathrm{D}-\mathrm{H} \cdots \mathrm{A}\left({ }^{\circ}\right)$ \\
\hline $\mathrm{O}(130)-\mathrm{H}(130) \cdots \mathrm{N}(1)$ & $0.97(2)$ & $1.67(2)$ & $2.5701(17)$ & $152.5(19)$ \\
$\mathrm{O}(430)-\mathrm{H}(430) \cdots \mathrm{N}(4)$ & $0.93(2)$ & $1.75(2)$ & $2.6112(18)$ & $151.8(18)$ \\
\hline
\end{tabular}

\section{REFERENCES}

1.- J. P. Costes, F.Dahan, B.Donnadieu, M. I.Fernández-García, M. J.Rodriguez-Doutón, Dalton Trans., 2003, 3776-3779.

2.- M. R.Bermejo, M. I.Fernández, E.Gómez-Fórneas, A.González-Noya, M.Maneiro, R.Pedrido, M. J.Rodriguez, Eur. J. Inorg. Chem., 2007, 3789-3797. 